Revue québécoise de psychologie

Wuevécécolise

\title{
ATTITUDES ET COMPORTEMENTS ALIMENTAIRES INAPPROPRIÉS ET CARACTÉRISTIQUES PSYCHOSOCIALES DES SPORTIFS : COMPARAISON ENTRE DEUX NIVEAUX DE PRATIQUE SPORTIVE \\ INAPPROPRIATE EATING ATTITUDES AND BEHAVIORS AND ATHLETES' PSYCHOSOCIAL CHARACTERISTICS: COMPARISON BETWEEN TWO LEVELS OF SPORT PRACTICE
}

\author{
Maud Bonanséa, Johana Monthuy-Blanc, Annie Aimé, Pierre Therme et \\ Christophe Maïano
}

Volume 37, numéro 1, 2016

LES TROUBLES DE LA CONDUITE ALIMENTAIRE

EATING DISORDERS

URI : https://id.erudit.org/iderudit/1040103ar

DOI : https://doi.org/10.7202/1040103ar

Aller au sommaire du numéro

Éditeur(s)

Revue québécoise de psychologie

ISSN

2560-6530 (numérique)

Découvrir la revue

Citer cet article

Bonanséa, M., Monthuy-Blanc, J., Aimé, A., Therme, P. \& Maïano, C. (2016). ATTITUDES ET COMPORTEMENTS ALIMENTAIRES INAPPROPRIÉS ET CARACTÉRISTIQUES PSYCHOSOCIALES DES SPORTIFS : COMPARAISON ENTRE DEUX NIVEAUX DE PRATIQUE SPORTIVE. Revue québécoise de psychologie, 37(1), 39-60. https://doi.org/10.7202/1040103ar
Résumé de l'article

L'objectif de cette étude est de comparer les attitudes et comportements alimentaires inappropriés (ACAI) ainsi que les caractéristiques psychosociales anxiété physique sociale, perceptions du soi physique, pression perçue pour être mince - entre deux niveaux de pratique sportive (intensive et de loisir). Cent quarante-six adolescents français ont rempli un ensemble de questionnaires. Les résultats indiquent que, comparativement aux sportifs qui s'entraînent en loisir, les sportifs de niveau intensif sont plus nombreux à avoir recours à des ACAI, ils ressentent également plus d'insatisfaction corporelle et d'anxiété physique sociale, ils sont moins satisfaits de leur apparence physique et ressentent plus de pression pour mincir. 


\section{ATTITUDES ET COMPORTEMENTS ALIMENTAIRES INAPPROPRIÉS ET CARACTÉRISTIQUES PSYCHOSOCIALES DES SPORTIFS : COMPARAISON ENTRE DEUX NIVEAUX DE PRATIQUE SPORTIVE ${ }^{1}$}

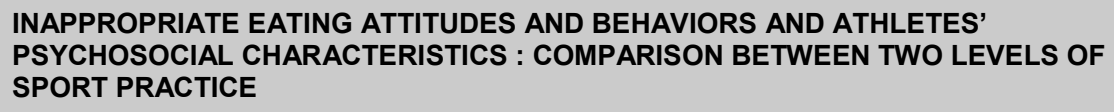

Maud Bonanséa ${ }^{2}$

Université du Québec à Trois-Rivières

Annie Aimé

Université du Québec en Outaouais

Christophe Maïano
Johana Monthuy-Blanc

Université du Québec à Trois-Rivières

Pierre Therme

Aix-Marseille Université

\section{INTRODUCTION}

L'activité physique est reconnue pour représenter un facteur de protection pour la santé mentale et physique en population générale (Cox, 2007). Cependant, en contexte de performance sportive, lorsqu'elle est pratiquée de manière intensive, l'activité physique peut être associée à des préoccupations élevées par rapport à l'alimentation et au poids et peut s'accompagner de comportements de restrictions alimentaires plus fréquents et plus nombreux (Chatterton \& Petrie, 2013). En effet, 25,5\% des sportifs comparativement à $15 \%$ de la population générale présentent des attitudes et des comportements alimentaires inappropriés (ACAI) (Ackard, Fulkerson, \& Neumark-Sztainer, 2007; Greenleaf, Petrie, Carter, \& Reel, 2009). En fait, les sportifs présentent des caractéristiques biopsychosociales et comportementales pouvant mener à des troubles du comportement alimentaire (TCA) tels que définis dans la cinquième édition du Manuel Diagnostique et Statistiques des troubles mentaux (DSM-5, APA, 2014). Plus précisément, ces caractéristiques peuvent être conceptualisées le long d'un continuum d'ACAl qui semble particulièrement indiqué dans le cas des sportifs (Sundgot-Borgen \& Torstveit, 2010).

Ce continuum illustre l'approche dimensionnelle des TCA et varie d'un état dit normal à un état clinique en passant par un état sous-clinique (Torstveit, Rosenvinge, \& Sundgot-Borgen, 2008; Tylka \& Subich, 2003).

1 L'équipe de recherche remercie les sportifs qui ont participé à l'étude ainsi que les entraîneurs qui ont accepté que cette étude soit conduite dans leur club sportif.

2. Adresse de correspondance: Département des sciences de l'éducation, C.P. 500, Trois-Rivières (QC), G9A 5H7. Téléphone: 819-376-5011, poste 3648. Courriel: maud.bonansea@uqtr.ca 
Des caractéristiques biopsychosociales et des comportements alimentaires sains correspondent à l'une des extrémités du continuum des ACAl, alors que des caractéristiques biopsychosociales et des comportements pathogènes problématiques, synonymes des TCA - tels que l'anorexie mentale, la boulimie et l'hyperphagie boulimique - illustrent l'autre extrémité (Johnson, 1994; Leung, Geller, \& Katzman, 1996; Sundgot-Borgen \& Torstveit, 2010). Ce continuum est présenté en Figure 1. Appliquée aux sportifs, l'extrémité de ce continuum qui s'apparente aux TCA peut correspondre à des troubles encore non reconnus dans le DSM-5 (APA, 2014), soit l'anorexie sportive ou athlétique ou encore une anorexie inversée ou bigorexie (Deimel \& Dunlap, 2012; Grieve, 2007). L'anorexie sportive présente plusieurs caractéristiques semblables à l'anorexie mentale, telles que la diminution significative du poids et la présence de méthodes de contrôle pondéral. Si son association à une distorsion de l'image corporelle est rare, la recherche d'un ratio poids/performance optimal la caractérise (Deimel \& Dunlap, 2012). La bigorexie, quant à elle, se définit par une prise pondérale relative à de l'hypertrophie musculaire, et ce, quels que soit les risques de santé encourus (Mosley, 2009). En d'autres termes, ces sportifs se perçoivent comme étant trop minces et trop chétifs et sont, par conséquent, dans une quête de l'augmentation de la masse musculaire, en utilisant dans certains cas des stéroïdes anabolisants (Mosley, 2009).

Entre ces deux extrémités (voir Figure 1), différents ACAl adoptés par les sportifs peuvent être observés. II s'agit par exemple des régimes chroniques, des jeûnes, de l'utilisation de coupe-faim ou de laxatifs, du recours à des vomissements provoqués ou encore de l'exercice physique excessif, des entraînements en combinaison de sudation ou des crises de suralimentation après des compétitions (Chatterton \& Petrie, 2013; Monthuy-Blanc \& Bonanséa, 2014; Monthuy-Blanc et al., 2010). Ces comportements sont associés à plusieurs caractéristiques biopsychosociales telles que le poids, le sexe, la pression perçue pour être mince, le surinvestissement lié à la réussite des parents ou la pression de performance, les affects négatifs, le perfectionnisme, l'anxiété physique sociale, l'insatisfaction corporelle, mais aussi les faibles perceptions du soi physique (Sundgot-Borgen et al., 2013). Notons que les perceptions du soi physique incluent: l'estime globale de soi, la valeur physique perçue, la condition physique, la compétence sportive, l'apparence physique et la force physique (Fox, 2000). Selon certaines études s'intéressant aux relations entre ces variables, les caractéristiques biopsychosociales peuvent expliquer, voire prédire les ACAl chez les sportifs (Brunet \& Sabiston, 2009; Monthuy-Blanc, Maïano, Morin, \& Stephan, 2012; Thompson \& Chad, 2002). 


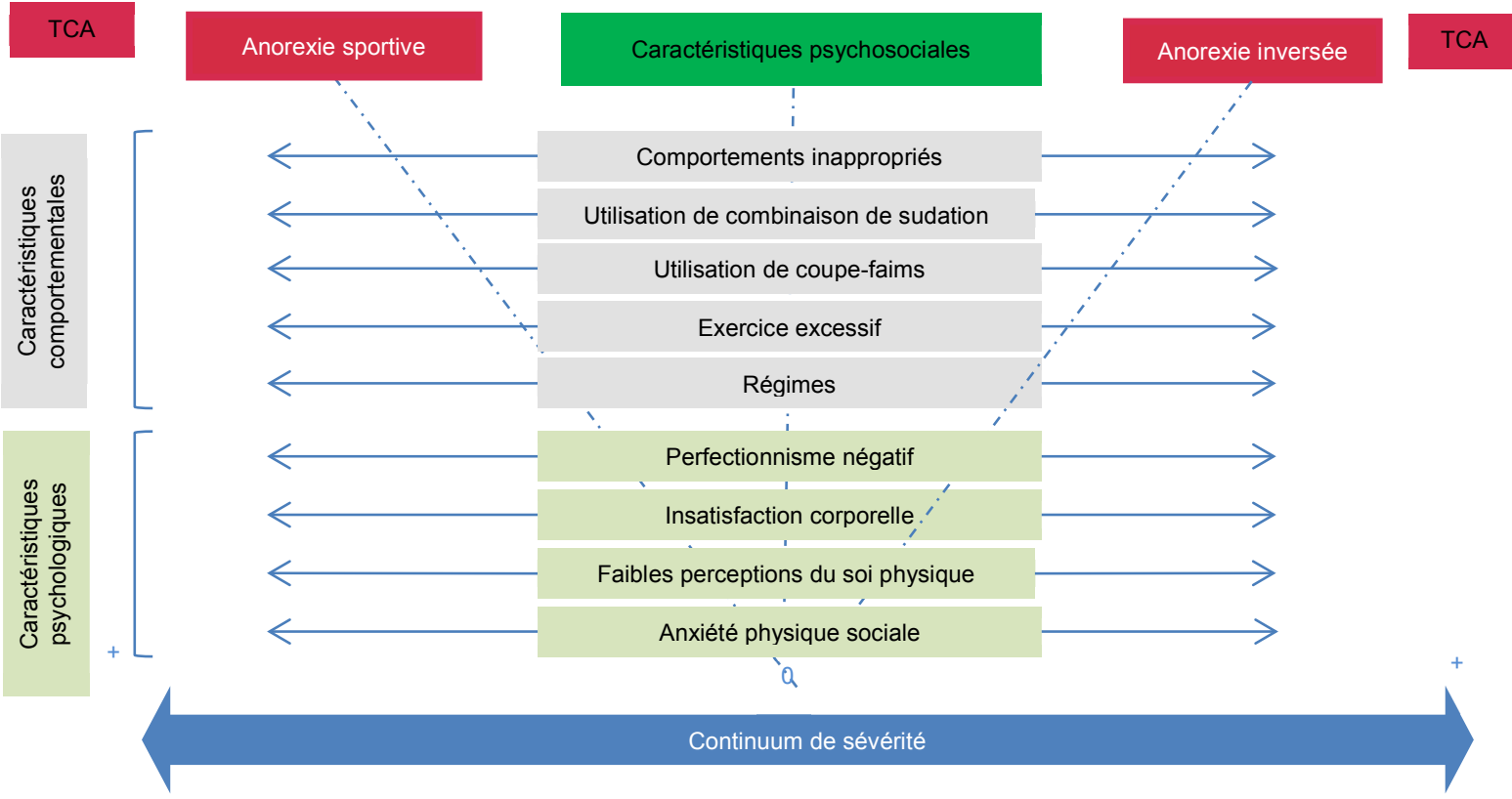

Figure 1. Continuum des attitudes et comportements alimentaires inappropriés chez le sportif, inspiré de Koszewski, Chopak et Buxton, 1997 
Plusieurs variables peuvent amener un sportif à se situer à différents niveaux de sévérité sur le continuum des ACAI, dont le type de pratique sportive, le niveau de pratique sportive ainsi que le sexe et l'âge des sportifs. Premièrement, concernant le type de pratique sportive, depuis les années 1990 plusieurs auteurs ont suggéré des classifications de pratiques sportives, allant de deux à six catégories, pouvant participer au développement d'ACAl chez le sportif (Currie, 2010; Sundgot-Borgen, 1994; Sundgot-Borgen \& Torstveit, 2004). Currie (2010) propose pour sa part une classification qui regroupe trois catégories de pratiques sportives selon lesquelles le poids corporel influence la performance sportive. Cet auteur regroupe des pratiques sportives dites (a) « longilignes » comme la gymnastique, qui nécessitent une forme corporelle particulière pour répondre aux performances techniques et artistiques (b) "d'endurance " ou "d'efficience » comme la natation ou la course à pied, au sein desquelles les sportifs les plus musclés sont à risque d'être perçus comme plus lents, (c) "à catégorie de poids " comme le judo, imposant un ratio poids/puissance optimal pour battre son adversaire (Currie, 2010). Selon le continuum de sévérité des ACAI décrits précédemment, chez les sportifs qui évoluent dans des pratiques sportives dites longilignes et d'endurance, les taux de prévalences d'ACAl se situent entre : $22 \%$ et $62 \%$ en gymnastique, $38,7 \%$ en escalade et $3,6 \%$ à $14,4 \%$ en natation (Beals, 2004). Dans des pratiques sportives à catégories de poids, ces taux se situent autour de 30 \% chez les sportives (Sundgot-Borgen et al., 2013).

Deuxièmement, concernant le niveau de pratique, celui-ci dépend principalement de plusieurs critères incluant la fréquence et le volume des entraînements, la présence ou l'absence de compétition ainsi que l'ancienneté de pratique. La littérature scientifique oppose le plus souvent le niveau de pratique dit " de loisir » à celui dit « intensif » (Kong \& Harris, 2014; Martinsen \& Sundgot-Borgen, 2013). Les sportifs de niveau loisir pratiquent entre une et six heure(s) par semaine. Ils le font dans une visée hédonique, sans qu'il y ait nécessairement présence d'entraînement régulier et de compétition (Fortes, Kakeshita, Almeida, Gomes, \& Ferreira, 2014). De plus, leur engagement dans la pratique sportive est généralement récent, allant d'un à deux ans. À l'inverse, les sportifs de niveau intensif s'entraînent fréquemment au-delà de huit heures par semaine, dans une visée compétitive, le plus souvent nationale, voire internationale (Sundgot-Borgen, 1994; Sundgot-Borgen \& Tortsveit, 2004). Ce sont des sportifs qui ont commencé à s'entraîner dès leur plus jeune âge, c'est-à-dire qu'ils pratiquent leur sport depuis cinq à dix ans dans certains cas (Sundgot-Borgen \& Tortsveit, 2004). Selon le continuum de sévérité des ACAI, Martinsen, Bratland-Sanda, Eriksson et SundgotBorgen (2010) rapportent que $47 \%$ des sportives de niveau intensif présentent des ACAl. De plus, $51,6 \%$ des sportifs inscrits aux compétitions nationales auraient recours à des comportements 
compensatoires inappropriés, tels que l'exercice physique excessif pour perdre du poids pouvant alors s'apparenter à une hyperactivité physique (Chatterton \& Petrie, 2013).

Troisièmement, l'âge et le sexe des sportifs peuvent également amener le sportif à se situer à différents niveaux sur le continuum des ACAI. Selon Fortes et ses collaborateurs (2014), les jeunes filles sportives âgées de 10 à 19 ans présentent davantage d'ACAl que les garçons sportifs de la même tranche d'âge (respectivement $18,1 \%$ et $14,4 \%$ ). Chez les jeunes sportives, le développement des attributs sexuels secondaires lors de la puberté peut être perçu comme un obstacle à la performance, ce qui pourrait ensuite donner lieu à des préoccupations envers l'image corporelle et éventuellement conduire au développement d'ACAI (Torstveit et al., 2008). Par ailleurs, les sportifs qui ont commencé à s'entraîner très tôt, soit en moyenne à 11,2 ans, sont plus vulnérables aux ACAI (Hoek, 2006; Lanfranchi, Maïano, Morin \& Therme, 2014).

Plusieurs études se sont intéressées à comparer de façon unidimensionnelle à la fois les caractéristiques psychosociales (c.-à-d. individuelles, sociales et culturelles) et comportementales chez des sportifs engagés dans une pratique et considérés à risque de développer des ACAl, en fonction de leur niveau de pratique (Gapin \& Kearns, 2013; Haase \& Prapavessis, 2001; Kong \& Harris, 2014; Martinsen et al., 2010). Sur le plan des ACAl, les études présentent des résultats très variés. L'étude de Turgeon, Meilleur et Blondin (2015) montre qu'un groupe d'adolescentes sportives pratiquant principalement la nage synchronisée et le patinage artistique à un niveau intensif ne présentent pas plus d'ACAl que les jeunes filles peu ou pas sportives. D'autres auteurs rapportent que les sportifs de niveau loisir présentent plus de comportements alimentaires inappropriés que les sportifs de niveau intensif (Darcy, Hardy, Lock, Hill, \& Peebles, 2013). Au final, plusieurs études suggèrent que les sportifs de niveau intensif ont plus souvent recours aux jeûnes, aux vomissements provoqués et à l'exercice physique excessif pour perdre du poids, comparé aux sportifs de niveau loisir (Petrie, Greenleaf, Reel, \& Carter, 2008). Des études font écho à ces résultats en montrant que les sportifs intensifs présentent des scores plus élevés que les sportifs de niveau loisir à l'échelle de boulimie du test des attitudes alimentaires (EAT-26; Fortes et al., 2014), et qu'ils rapportent davantage d'insatisfaction corporelle que les sportifs de niveau loisir (Kong \& Harris, 2014). En ce qui a trait aux caractéristiques psychosociales, Haase et Prapavessis (2001) rapportent que les sportifs de niveau intensif et de niveau loisir éprouvent le même niveau d'anxiété physique sociale. Concernant la pression perçue pour mincir, les études démontrent que les sportifs de niveau intensif ressentent plus de pression pour mincir et perdre du poids de la part de leurs 
coéquipiers et de leurs entraîneurs (Byrne \& McLean, 2002; Peden, Stiles, Vandehey, \& Diekhoff, 2008; Smolak, Murnen, \& Ruble, 2010).

Peu d'études portant sur les sportifs ont utilisé le continuum des ACAI afin de distinguer différents niveaux de pratiquants sur le plan des caractéristiques comportementales et psychosociales. Les quelques études disponibles ne cherchent généralement qu'à évaluer une seule de ces caractéristiques selon le sexe ou encore selon un type de pratique sportive. Lorsque plusieurs de ces caractéristiques sont évaluées, l'objectif est de déterminer la prévalence des TCA en excluant tout état intermédiaire, comme le propose le continuum des ACAI (Toro et al., 2005, Torstveit et al., 2008). Or, chez les sportifs, la conceptualisation des ACAI le long d'un continuum permet de prendre en considération des comportements liés à leur pratique sportive qui ne satisfont pas nécessairement les critères associés à un TCA particulier. L'objectif de la présente étude est de comparer les ACAl et les caractéristiques psychosociales entre des sportifs de niveau intensif et des sportifs de niveau loisir. La première hypothèse suppose que les sportifs de niveau intensif obtiennent des scores plus élevés sur la mesure de boulimie, de l'insatisfaction corporelle et du désir de minceur et qu'ils seront plus nombreux à avoir recours à des comportements compensatoires inappropriés. Concernant les caractéristiques psychosociales, la seconde hypothèse postule un niveau plus élevé d'anxiété physique sociale et de pression en lien avec le poids et l'apparence ainsi qu'un niveau plus élevé des perceptions du soi physique chez les sportifs de niveau intensif que chez les sportifs de loisir.

\section{MÉTHODE}

\section{Participants et procédure}

L'échantillon est composé de 146 adolescents et jeunes adultes français, soit 74 garçons et 72 filles, âgés de 13 à 20 ans et engagés dans une pratique sportive intensive $(n=88)$ ou de loisir $(n=58)$, du type athlétisme, nage synchronisée, gymnastique, judo et taekwondo. Dans cet échantillon, une pratique sportive intensive correspond à un volume moyen d'entraînement hebdomadaire de 13 heures alors qu'une pratique sportive de loisir correspond en moyenne à 4 heures d'entraînement hebdomadaire. Le Tableau 1 présente les données anthropométriques des sportifs et les données liées à la pratique sportive.

Les participants ont été recrutés dans deux sites différents, à savoir des pôles France pour les sportifs de niveau intensif et des clubs civils pour les sportifs considérés de loisir. Cette étude a reçu l'accord du comité éthique de l'université de la Méditerranée Aix-Marseille II. Pour les 
RQP, 37(1)

Tableau 1

Données anthropométriques et données liées à la pratique sportive

\begin{tabular}{|c|c|c|c|c|c|c|}
\hline \multirow{2}{*}{$\begin{array}{l}\text { Données } \\
\text { anthropométriques/ } \\
\text { sportives }\end{array}$} & \multicolumn{2}{|c|}{$\begin{array}{l}\text { Intensif } \\
(n=88)\end{array}$} & \multicolumn{2}{|c|}{$\begin{array}{l}\text { Loisir } \\
(n=58)\end{array}$} & \multicolumn{2}{|c|}{$\begin{array}{c}\text { Total } \\
(n=146)\end{array}$} \\
\hline & \multicolumn{2}{|c|}{$M \pm E T$} & \multicolumn{2}{|r|}{$M \pm E T$} & \multicolumn{2}{|c|}{$M \pm E T$} \\
\hline Âge (années) & \multicolumn{2}{|c|}{$17,06 \pm 1,26$} & \multicolumn{2}{|c|}{$16,33 \pm 1,72$} & \multicolumn{2}{|c|}{$16,77 \pm 1,50$} \\
\hline $\operatorname{IMC}\left(\mathrm{kg} / \mathrm{m}^{2}\right)$ & \multicolumn{2}{|c|}{$22,23 \pm 4,19$} & \multicolumn{2}{|c|}{$19,75 \pm 2,01$} & \multicolumn{2}{|c|}{$21,24 \pm 3,69$} \\
\hline Catégorie de poids & $\mathrm{N}$ & $\%$ & $\mathrm{~N}$ & $\%$ & $\mathrm{~N}$ & $\%$ \\
\hline Insuffisance pondérale & 12 & $13,64 \%$ & 13 & $22,41 \%$ & 25 & $17,12 \%$ \\
\hline Poids normal & 55 & $62,50 \%$ & 44 & $75,86 \%$ & 99 & $67,81 \%$ \\
\hline Surpoids & 17 & $19,32 \%$ & 1 & $1,72 \%$ & 18 & $12,33 \%$ \\
\hline Obésité & 4 & $4,55 \%$ & 0 & - & 4 & $2,74 \%$ \\
\hline \multicolumn{7}{|l|}{$\begin{array}{l}\text { Types de pratiques } \\
\text { sportives }\end{array}$} \\
\hline Judo & 50 & $56,82 \%$ & 18 & $31,03 \%$ & 68 & $46,58 \%$ \\
\hline Taekwondo & 11 & $12,05 \%$ & 13 & $22,41 \%$ & 24 & $16,44 \%$ \\
\hline Gymnastique & 6 & $6,82 \%$ & 7 & $12,07 \%$ & 13 & $8,90 \%$ \\
\hline Nage synchronisée & 5 & $5,68 \%$ & 6 & $10,35 \%$ & 11 & $7,53 \%$ \\
\hline Athlétisme & 16 & $1,82 \%$ & 14 & $24,14 \%$ & 30 & $20,55 \%$ \\
\hline Niveau de pratique & \multicolumn{2}{|c|}{$M \pm E T$} & \multicolumn{2}{|r|}{$M \pm E T$} & \multicolumn{2}{|c|}{$M \pm E T$} \\
\hline $\begin{array}{l}\text { Ancienneté de pratique } \\
\text { (années) }\end{array}$ & \multicolumn{2}{|c|}{$10,00 \pm 3,64$} & \multicolumn{2}{|c|}{$4,34 \pm 2,94$} & \multicolumn{2}{|c|}{$7,78 \pm 4,35$} \\
\hline $\begin{array}{l}\text { Temps entraînement } \\
\text { moyen (min) }\end{array}$ & \multicolumn{2}{|c|}{$2,11 \pm 0,49$} & \multicolumn{2}{|c|}{$1,95 \pm 0,57$} & \multicolumn{2}{|c|}{$2,05 \pm 0,53$} \\
\hline $\begin{array}{l}\text { Nombre d'entraînement } \\
\text { par semaine }\end{array}$ & \multicolumn{2}{|c|}{$6,40 \pm 2,25$} & \multicolumn{2}{|c|}{$2,24 \pm 0,47$} & \multicolumn{2}{|c|}{$4,75 \pm 2,71$} \\
\hline $\begin{array}{l}\text { Volume de pratique } \\
\text { sportive par semaine } \\
\text { (hrs) }\end{array}$ & \multicolumn{2}{|c|}{$13,61 \pm 6,05$} & & $, 44 \pm 1,99$ & \multicolumn{2}{|c|}{$9,96 \pm 6,62$} \\
\hline
\end{tabular}

Note : $M=$ Moyenne; E.T. = écart-type; $N=$ échantillon; $I M C=$ Indice de masse corporelle; $\min$ $=$ minutes; hrs = heures

participants mineurs (moins de 18 ans), le consentement à la participation de leurs parents a été obtenu en plus de leur propre consentement. Une lettre explicative de l'étude accompagnée d'un formulaire de consentement libre et éclairé a été remise à chacun des participants par l'entraîneur afin qu'ils puissent prendre connaissance de la recherche. Une fois le formulaire de consentement signé, l'entraîneur l'a ensuite transmis à la première auteure de cet article. Un code numérique connu seulement de la première auteure a été attribué à chaque participant afin d'assurer son anonymat. Des groupes de 10 à 12 sportifs ont été constitués afin que la passation des questionnaires autorapportés puisse avoir lieu dans des conditions optimales. Ces derniers ont été remplis sur les lieux de pratique 
pour l'ensemble des 146 participants, en présence de la première auteure seulement.

\section{Instruments de mesure}

Des données démographiques portant sur les caractéristiques des participants ainsi que sur leur pratique sportive ont été recueillies à l'aide d'un questionnaire d'informations générales. Ainsi, les participants ont euxmêmes rapporté leur âge, leur sexe, leur poids et leur taille. Ils ont aussi été questionnés sur leur type de pratique sportive, leur niveau de pratique (intensif ou de loisir), le volume et la fréquence de la pratique sportive au sein du club ou du pôle. Leur indice de masse corporelle (IMC) a été calculé en divisant leur poids en kilogramme par leur taille en mètre au carré $\left(\mathrm{kg} / \mathrm{m}^{2}\right)$. Des catégories d'IMC (insuffisance pondérale, poids normal, surpoids, obésité) ont ensuite été formées en tenant compte de l'âge et du sexe des participants, tel que proposé par Cole, Bellizzi, Flegal et Dietz (2000) et Cole, Flegal, Nicholls et Jackson (2007).

\section{Caractéristiques comportementales : les attitudes et comportements alimentaires inappropriés (ACAI)}

La présence et la sévérité des crises de suralimentation et des comportements compensatoires inappropriés ont été mesurées avec le Questionnaire for Eating Disorder Diagnosis (QEDD de Mintz, O'holloran, Mulholland et Schneider, 1997), validé en langue française par Callahan et ses collaborateurs (2003), sous le nom de QDTCA. Neuf caractéristiques comportementales relatives aux ACAI ont été évaluées à l'aide de 27 items, soit les crises de suralimentation, le recours aux vomissements provoqués, aux lavements, jeûnes, régimes, mâcher-recracher et à l'utilisation de laxatifs, coupe-faims et diurétiques. Trois items permettent d'évaluer chacun de ces comportements. Cet autoquestionnaire propose des modes de réponse à l'item variés : format « oui/non », choix multiple (durée et fréquence du comportement) ou une demande d'informations substantives (comme la taille, le poids, etc.). La consistance interne est présentée dans le Tableau 2.

Les ACAl ont été mesurés grâce à la version française courte à 24 items (EDI-A-24 de Maïano, Morin, Ninot, Monthuy-Blanc, Garbarino, \& Stephan, 2009) du Eating Disorders Inventory (Garner, Olmstead, \& Polivy, 1983). Dans le cadre de cette étude, seules les échelles de boulimie, insatisfaction corporelle et désir de minceur de l'EDI-A-24 ont été utilisées. Elles comportent chacune trois items auxquels les participants répondent en utilisant une échelle de Likert en six points allant de 5 (toujours) à 0 (jamais). La consistance interne est présentée dans le Tableau 2. 
RQP, 37(1)

Tableau 2

Caractéristiques comportementales et psychosociales pour les deux groupes de sportifs

\begin{tabular}{|c|c|c|c|c|c|c|c|}
\hline \multirow[t]{2}{*}{ Questionnaires } & \multirow[t]{2}{*}{$\alpha$} & \multicolumn{2}{|c|}{$\begin{array}{l}\text { Intensif } \\
(n=88)\end{array}$} & \multicolumn{2}{|c|}{$\begin{array}{l}\text { Loisir } \\
(\mathrm{n}=58)\end{array}$} & \multirow[t]{2}{*}{$F(1,145)$} & \multirow{2}{*}{$p$} \\
\hline & & $N$ & $\%$ & $N$ & $\%$ & & \\
\hline QDTCA & .70 & & & & & & \\
\hline Crises de suralimentation & .70 & 18 & $20,45 \%$ & 8 & $13,79 \%$ & 1.06 & \\
\hline Perte de contrôle & - & 7 & $7,95 \%$ & 3 & $5,17 \%$ & 0.03 & * \\
\hline Vomissements & .72 & & $2,27 \%$ & 0 & & 1.34 & \\
\hline Laxatifs & .74 & 4 & $4,55 \%$ & 0 & & 2.71 & \\
\hline Diurétiques & .61 & 1 & $1,14 \%$ & 0 & & 0.66 & \\
\hline Coupe-faim & .76 & 6 & $6,82 \%$ & 3 & $5,17 \%$ & 0 & \\
\hline Lavements & - & 0 & & 0 & & 0.16 & \\
\hline Jeûnes & .67 & 15 & $17,05 \%$ & 2 & $3,45 \%$ & 6.40 & \\
\hline Mâcher/recracher & .75 & 1 & $1,14 \%$ & 0 & & 0.65 & \\
\hline \multirow[t]{2}{*}{ Régimes } & .76 & 26 & $29,55 \%$ & 3 & $5,17 \%$ & 13.05 & *** \\
\hline & \multicolumn{3}{|c|}{$\mathrm{M} \pm \mathrm{ET}$} & \multicolumn{2}{|c|}{$\mathrm{M} \pm \mathrm{ET}$} & & \\
\hline \multicolumn{8}{|l|}{ Échelles de l'EDI-A-24 } \\
\hline Boulimie & .85 & \multicolumn{2}{|c|}{$6,37 \pm 3,02$} & \multicolumn{2}{|c|}{$3,10 \pm 3,04$} & 8.54 & *** \\
\hline Insatisfaction corporelle & .69 & \multicolumn{2}{|c|}{$7,44 \pm 3,90$} & \multicolumn{2}{|c|}{$5,51 \pm 3,90$} & 8.54 & *** \\
\hline Désir de minceur & .85 & \multicolumn{2}{|c|}{$5,17 \pm 2,95$} & \multicolumn{2}{|c|}{$2,59 \pm 3,68$} & 19.01 & * \\
\hline EAPS & .89 & \multicolumn{2}{|c|}{$16,28 \pm 6,98$} & \multicolumn{2}{|c|}{$12,10 \pm 5,56$} & 14.70 & $* * *$ \\
\hline \multicolumn{8}{|l|}{ Échelles de l'ISP } \\
\hline Estime globale de soi & .67 & \multicolumn{2}{|c|}{$4,22 \pm 1,10$} & \multicolumn{2}{|c|}{$4,27 \pm 1,10$} & 0.09 & \\
\hline Valeur physique & .87 & \multicolumn{2}{|c|}{$4,04 \pm 1,09$} & \multicolumn{2}{|c|}{$4,00 \pm 0,94$} & 0.05 & \\
\hline Condition physique & .70 & \multicolumn{2}{|c|}{$3,59 \pm 1,38$} & \multicolumn{2}{|c|}{$4,10 \pm 1,12$} & 5.80 & \\
\hline Compétence sportive & .61 & \multicolumn{2}{|c|}{$4,10 \pm 0,99$} & \multicolumn{2}{|c|}{$4,05 \pm 0,86$} & 0.10 & \\
\hline Apparence physique & .82 & \multicolumn{2}{|c|}{$4,13 \pm 1,03$} & \multicolumn{2}{|c|}{$4,69 \pm 0,84$} & 12.16 & * \\
\hline Force physique & .75 & \multicolumn{2}{|c|}{$3,39 \pm 1,23$} & & $\pm 0,90$ & 3.97 & \\
\hline Pression socioculturelle perçue & .89 & & & & & & \\
\hline $\begin{array}{l}\text { Pression de l'entraîneur } \\
\text { perçue pour }\end{array}$ & & & & & & & \\
\hline Être mince & & & $\pm 0,78$ & & $\pm 0,26$ & 6.60 & \\
\hline Faire un régime & & & $\pm 0,88$ & & $\pm 0,29$ & 13.20 & *** \\
\hline Perdre du poids & & & $\pm 0,89$ & & $\pm 0,29$ & 18.00 & *** \\
\hline
\end{tabular}


ACAl et niveau de pratique sportive

Tableau 2

Caractéristiques comportementales et psychosociales pour les deux groupes de sportifs (suite)

\begin{tabular}{|c|c|c|c|c|c|}
\hline Questionnaires & $\alpha$ & $\begin{array}{l}\text { Intensif } \\
(n=88)\end{array}$ & $\begin{array}{l}\text { Loisir } \\
(n=58)\end{array}$ & $F(1,145)$ & $p$ \\
\hline & & $\mathrm{M} \pm \mathrm{ET}$ & $\mathrm{M} \pm \mathrm{ET}$ & & \\
\hline $\begin{array}{l}\text { Pression des coéquipiers } \\
\text { perçue pour }\end{array}$ & .91 & & & & \\
\hline Être mince & & $0,14 \pm 0,43$ & $0,02 \pm 0,13$ & 4.10 & \\
\hline Faire un régime & & $0,32 \pm 0,74$ & $0,02 \pm 0,13$ & 9.50 & * \\
\hline Perdre du poids & & $0,21 \pm 0,65$ & $0,02 \pm 0,13$ & 11.20 & ** \\
\hline $\begin{array}{l}\text { Pression des parents perçue } \\
\text { pour }\end{array}$ & .92 & & & & \\
\hline Être mince & & $0,5 \pm 1,26$ & $0,47 \pm 1,38$ & 0.02 & \\
\hline Faire un régime & & $0,80 \pm 1,43$ & $0,26 \pm 0,83$ & 6.70 & \\
\hline Perdre du poids & & $0,81 \pm 1,52$ & $0,36 \pm 1,12$ & 3.60 & \\
\hline
\end{tabular}

Note. $\mathrm{M}=$ Moyenne; $\mathrm{E} . \mathrm{T}$ = écart-type; $\mathrm{N}=$ échantillon; $E A P S=$ Échelle d'anxiété physique sociale; ISP = Inventaire du soi physique; ${ }^{*} p<.05 ;{ }^{* *} p<.01 ;{ }^{* * *} p<.001$ en appliquant une correction de Bonferroni; $\alpha=$ alpha de Cronbach; $F=$ ANOVAs EDI-A-24 = Inventaire des TCA pour adolescents; QDTCA = Questionnaire Diagnostique des troubles du comportement alimentaire; EAPS= Échelle d'anxiété physique sociale; $\mathrm{x}^{2}$ : Chi-Carré;

\section{Caractéristiques psychosociales}

Le niveau d'anxiété physique sociale a été mesuré à l'aide de la Social Physical Anxiety Scale (SPAS de Hart, Leary, \& Rejeski, 1989) validée en français sous le nom d'Échelle d'anxiété physique sociale (EAPS, Maïano, Morin, Eklund, Monthuy-Blanc, Garbarino, \& Stephan, 2010). Dans ce questionnaire, chaque participant répond aux sept items par une échelle de Likert à cinq degrés croissants allant de 0 (pas du tout) à 5 (extrêmement). La consistance interne est présentée dans le Tableau 2.

Les perceptions du soi physique ont été évaluées à l'aide de la version très courte de l'Inventaire du soi physique (ISP) à 12 items de Maïano et ses collaborateurs (2008). Ce questionnaire mesure six dimensions du soi physique : l'estime globale de soi, la valeur physique perçue, l'endurance, la force, l'apparence physique et les compétences sportives. Les participants répondent à chacun des items en utilisant une échelle de Likert à six degrés croissants allant de 1 (pas du tout) à 6 (tout à fait). La consistance interne est présentée dans le Tableau 2.

La pression perçue pour être mince exercée par la famille et les amis du participant est mesurée à l'aide de la version française et modifiée de l'Échelle de pression socioculturelle perçue (Stice \& Agras, 1998). Les items retenus dans cette étude sont ceux qui s'appliquent le mieux au 
contexte sportif, soit ceux relatifs à la pression des coéquipiers, entraîneur et parents. Les participants répondent aux neuf items en utilisant une échelle de Likert à cinq degrés croissants allant de 0 (aucune pression) à 5 (beaucoup de pression). La consistance interne est présentée dans le Tableau 2

\section{Analyses}

La version 22 du logiciel SPSS a été utilisée pour les analyses. La normalité des distributions a été vérifiée au moyen des tests de ShapiroWilks et de Kolmogorov-Smirnov. Les comparaisons entre les groupes de sportifs ont été réalisées à l'aide de tests du Chi-Carré $\left(X^{2}\right)$ et d'analyses de variance (ANOVA univariées et ANCOVAs univariées). Alors que les Chi-Carré ont permis de comparer le pourcentage de sportifs (intensif ou de loisir) sur le plan des ACAI, les ANOVAs univariées ont été appliquées pour déterminer si les deux groupes de sportifs se distinguent sur les plans des caractéristiques psychosociales. Une correction de Bonferroni a été appliquée pour contrôler l'inflation des probabilités de commettre une erreur de type 1, lors des tests d'ANOVAs et Chi-Carrés. Pour l'EDI-A24, le seuil de signification est fixé à ${ }^{*} p<.0167(.05 / 3)$, pour le QDTCA et l'Échelle de pression socioculturelle perçue à ${ }^{*} p<.0056(.05 / 9)$ et enfin pour l'ISP le seuil de signification est fixé à ${ }^{*} p<.0083(.05 / 6)^{1}$. Les ANCOVAs ont été réalisées pour les données relatives aux attitudes et comportements boulimiques, l'insatisfaction corporelle et le désir de minceur ainsi que pour les caractéristiques psychologiques à savoir les perceptions du soi physique, l'anxiété physique sociale, afin de contrôler la variable âge dans le but de palier la disparité de notre échantillon.

\section{RÉSULTATS}

\section{Caractéristiques descriptives de l'échantillon}

Concernant les données anthropométriques, les sportifs de l'échantillon sont en moyenne âgés de 16,77 ans (ÉT $=1,50$ ) et leur IMC moyen est de $21,24 \mathrm{~kg} / \mathrm{m}^{2}$ (ÉT = 3,69). Au total, 25 sportifs sont en insuffisance pondérale, soit 12 sportifs de niveau intensif $(19,39 \%)$ et 13 sportifs de niveau loisir (1,72\%). Quatre-vingt-dix-neuf sportifs sont de poids normal, soit 55 sportifs de niveau intensif $(62,50 \%)$ et 44 sportifs de niveau loisir $(75,86 \%)$. Dix-huit sportifs sont en surpoids, soit 17 sportifs de niveau intensif $(19,39 \%)$ et un sportif de niveau loisir (1,72\%). Enfin,

1. Après l'application de la correction de Bonferroni, les seuils de significations sont fixés pour l'EDI-A24, le seuil de signification est fixé à * $p<.0167(.05 / 3),{ }^{* *} p<.0033(.01 / 3)$ et ${ }^{* * \star} p<.0003(.001 / 3)$, pour le QDTCA et l'échelle de pression socioculturelle perçue à ${ }^{*} p<.0056(.05 / 9){ }^{* *} p<.0011(.01 / 3)$ et ${ }^{* *} p<.0001(.001 / 3)$ et enfin, pour l'ISP, le seuil de significativité est fixé à ${ }^{*} p<.0083(.05 / 6){ }^{* \star} p<.0017(.01 / 6)$ et ${ }^{* \star *} p<.0002(.001 / 6)$. 
quatre sportifs $(2,74 \%)$ sont considérés comme obèses, tous s'entraînant de façon intensive dans des pratiques sportives de prise de poids.

Concernant la pratique sportive, $56,82 \%$ des sportifs de niveau intensif pratiquent le judo, $12,05 \%$ le taekwondo, 6,82 \% la gymnastique, $5,68 \%$ la natation synchronisée et 1,82 \% l'athlétisme. Ils pratiquent en moyenne depuis 10 ans (ÉT $=3,64$ ) et s'entraînent en moyenne 13,61 heures par semaine (ÉT $=6,05)$. Les sportifs de loisir quant à eux sont $31,03 \%$ à pratiquer le judo, $22,41 \%$ le taekwondo, $12,07 \%$ la gymnastique, $10,35 \%$ la nage synchronisée et $24,14 \%$ l'athlétisme. Ils pratiquent en moyenne depuis 4,34 ans (ÉT $=2,94$ ) et s'entraînent en moyenne 4,44 heures par semaine (ÉT $=1,99$ ).

Attitudes et comportements alimentaires inappropriés (ACAI)

Concernant les comportements alimentaires inappropriés relevés à l'aide du QDTCA, les résultats du test de $\chi^{2}$ révèlent que, comparativement aux sportifs de niveau loisir, les sportifs de niveau intensif sont plus nombreux à rapporter des crises de suralimentation accompagnées d'un sentiment de perte de contrôle et à avoir recours à des comportements compensatoires inappropriés. Les fréquences sont présentées dans le Tableau 2. En ce qui a trait aux moyens de contrôler son poids, une seule différence significative est constatée entre les sportifs des deux niveaux de pratique sur le plan du recours aux régimes $\left(x^{2}=13.05, p<.001\right)$.

Sur le plan des échelles relatives aux attitudes et comportements alimentaires évaluées par l'EDI-A-24, les résultats montrent que les sportifs de niveau intensif obtiennent des scores significativement plus élevés que les sportifs de niveau loisir aux échelles de boulimie $[F(1,145)=8.54, p<.001]$, d'insatisfaction corporelle $[F(1,145)=8.54$, $p<.001]$ et de désir de minceur $[F(1,145)=19.01, p<.05]$. Les résultats des ANCOVAs montrent qu'au-delà de l'effet des variables de contrôle comme l'âge, le fait d'être un sportif de niveau intensif ou non a un effet significatif sur les comportements boulimiques $[F(1,145)=20.94, p<.001]$.

\section{Caractéristiques psychosociales}

Les ANOVAs réalisées indiquent un niveau d'anxiété physique sociale significativement plus élevé chez les sportifs de niveau intensif comparativement aux sportifs de niveau loisir $[F(1,145)=14.70, p<.001]$. De plus, les sportifs de niveau intensif présentent des scores moyens significativement plus faibles que les sportifs de niveau loisir à l'Échelle d'apparence physique perçue $[F(1,45)=12.16, p<.05]$.

L'analyse des résultats des ANOVAs indique également que, de façon générale, les sportifs de niveau intensif perçoivent plus de pression de la 
part de leur entraîneur pour faire un régime $[F(1,145)=13.20, p<.001]$ ou perdre du poids $[F(1,145)=18.00, p<.001]$ que les sportifs de niveau loisir. Ils perçoivent aussi de la part de leurs coéquipiers plus de pression pour faire un régime $[F(1,145)=9.50, p<.05]$ et perdre du poids $[F(1,145)=11.20, p<.01]$ que les sportifs de niveau loisir. Les analyses de variance n'indiquent pas de différence significative entre les deux groupes concernant la pression perçue de la part des parents pour faire un régime, perdre du poids ou être mince (voir Tableau 2).

\section{DISCUSSION}

L'objectif de la présente étude était de comparer les ACAI et certaines caractéristiques psychosociales entre deux groupes de sportifs, soit ceux de niveau intensif et ceux de niveau loisir. Si aucun résultat statistiquement significatif n'est relevé, le niveau de pratique sportive varie en fonction de l'âge, de l'ancienneté, de la fréquence et du type de pratique sportive, mais pas en fonction du sexe dans cet échantillon. Ainsi, les analyses descriptives semblent indiquer que les sportifs de niveau loisir sont plus jeunes et pratiquent leur sport depuis moins longtemps. Dans cette étude, les sportifs de niveau intensif sont plus susceptibles de pratiquer un sport nécessitant un poids et une masse musculaire plus élevés. En effet, la masse maigre (densité osseuse et musculaire) étant une fois et demie plus lourde que la masse graisseuse (Mosley, 2009), il semble justifié que les sportifs de niveau intensifs, de par leur investissement dans des types de sport de combat tels que le judo et le taekwondo, pèsent « plus lourd » que les sportifs de niveau loisir. Ainsi, les différences observées relatives à l'IMC pourraient être le reflet d'une pratique spécifique d'un type de sport ou d'une discipline sportive plutôt que d'un niveau de pratique. En effet, une distinction entre les différents types de pratiques sportives peut être faite, sachant que dans les pratiques sportives longilignes (comme la gymnastique), les enjeux de performance liés à l'IMC diffèrent étant donné l'absence de catégorie de poids. Cette donnée pose alors question quant à l'utilisation de l'IMC pour évaluer la caractéristique biologique du sportif. Par ailleurs, les résultats montrent que les sportifs de niveau intensif présentent un volume et une fréquence des entraînements supérieurs aux sportifs de niveau loisir. Ce dernier résultat semble intéressant concernant l'hypothèse d'une possible hypertrophie musculaire, pouvant être associée à la bigorexie. Les sportifs de niveau intensif peuvent être plus à risque de porter un engagement et un dévouement plus grand à leur pratique sportive et de ne pas se trouver suffisamment musclés pour performer. Ces caractéristiques se retrouvent chez les personnes atteintes de dysmorphie musculaire. Ces personnes peuvent ressentir de la honte face à leur corps, qu'ils jugent trop petit ou pas assez musclé, et ainsi recourir à des méthodes de gain musculaire dangereuses comme la prise de 
suppléments ou de psychotropes (c.-à-d., stéroïdes anabolisants, Mosley, 2009).

Notre première hypothèse, qui supposait que les sportifs de niveau intensif obtiennent des scores plus élevés aux échelles de boulimie, d'insatisfaction corporelle et de désir de minceur, que les sportifs de niveau loisir, et qu'ils soient plus nombreux à avoir recours à des ACAl est confirmée. Plus précisément dans notre échantillon, les sportifs de niveau intensif obtiennent à l'échelle de boulimie des scores en moyenne deux fois supérieurs aux sportifs de niveau loisir et ils sont cinq fois plus nombreux à rapporter des comportements de restriction alimentaire comme les régimes. Ces résultats vont dans le sens des études de Chatterton et Petrie (2013) et de Martinsen et ses collaborateurs (2010). Tel qu'indiqué par Bratland-Sanda et Sundgot-Borgen (2013), il est possible que les sportifs de niveau intensif aient tendance à avoir recours à des comportements compensatoires inappropriés dans le but d'obtenir une meilleure condition physique et de réaliser ainsi de meilleures performances. De ce fait, un volume et une fréquence de pratique sportive plus élevés sont associés à des comportements de contrôle du poids se situant à un niveau plus élevé sur le continuum des ACAl et impliquant des restrictions alimentaires plus importantes. Chatterton et Petrie (2013) suggèrent en ce sens que les sportifs de niveau intensif, de par le type de pratique sportive dans lequel ils sont engagés, sont plus à risque de juger négativement leur corps et de chercher à le modifier. Les présents résultats vont dans ce sens étant donné que les sportifs de niveau intensif présentent également une plus grande insatisfaction corporelle et un plus grand désir de minceur. Notons également que les pratiques sportives comme le judo et le taekwondo peuvent être associées à des restrictions alimentaires combinées à des méthodes excessives de perte de poids périodiques en fonction des catégories de poids imposées lors des compétitions (Currie 2010; Sundgot-Borgen \& Torstveit, 2010). Ainsi, il est probable que les sportifs de niveau intensif optent pour des restrictions alimentaires afin de modifier leur corps, dans le but d'atteindre de meilleures performances sportives (de Bruin, Oudejans, Bakker, \& Woertman, 2011, Hua \& Braddock, 2008). Or, la recherche d'une meilleure performance peut conduire à une chronicisation des comportements de restriction alimentaire, représentés par les régimes qui, à leur tour, augmentent le risque d'avoir des crises de suralimentation, de recourir à des comportements compensatoires inappropriés, donc de développer, éventuellement, un TCA (Fairburn, 2008). Dans notre échantillon, les différences d'ACAl observées entre les deux niveaux de sportifs peuvent également indiquer un risque plus élevé de développer une certaine forme de dysmorphie musculaire chez les sportifs de plus haut niveau, pour lesquels le volume et la fréquence de pratique d'un sport sont plus importants. 
La deuxième hypothèse concernant les caractéristiques psychosociales se voit partiellement confirmée par nos résultats. Premièrement, les sportifs de niveau intensif et de niveau loisir se perçoivent de la même façon sur le plan de l'estime globale de soi, de la valeur physique perçue, de la condition physique perçue, de la compétence sportive perçue ainsi que de la force physique perçue. La pratique sportive permettant d'améliorer ces perceptions (Grandmontagne \& Fernández, 2002), il est possible de se questionner sur les raisons pouvant expliquer que des sportifs qui pratiquent plus et qui sont davantage reconnus comme des experts dans leur pratique sportive n'aient pas une meilleure perception d'eux-mêmes, comparée à des sportifs de loisir. Or, dans l'échantillon actuel, les sportifs de niveau intensif sont plus insatisfaits de leur corps et entretiennent une perception de leur apparence physique plus négative, comparativement aux sportifs de niveau loisir. Ces résultats pourraient être en partie expliqués par le fait que les sportifs intensifs sont généralement satisfaits de leur corps dans un contexte social, mais insatisfaits dans le domaine sportif de performance (de Bruin et al., 2011). Ces derniers peuvent se fixer des objectifs plus élevés en termes de pratique sportive (versus en contexte social) et ces objectifs ne peuvent pas toujours être atteints. Ainsi, cette pression de performance pourrait venir modérer l'influence protectrice de la pratique sportive chez ces sportifs (Cox, 2007). De plus, ces résultats supposent que l'insatisfaction corporelle et le désir de minceur observés chez les sportifs de niveau intensif puissent être liés à des remarques négatives faites par les entraîneurs ou les coéquipiers (Monthuy-Blanc et al., 2010). En contexte de pratique sportive intensive, il est probable que les mauvaises ou moins bonnes performances réalisées par les sportifs soient attribuées à une gestion du poids jugée inadéquate ou encore au fait de ne pas posséder les bons attributs physiques pour bien réussir dans un sport donné (Kong \& Harris, 2014). Des remarques négatives sur l'apparence physique et des comparaisons entre coéquipiers sur le plan de la morphologie ou du poids peuvent par ailleurs contribuer à favoriser le développement d'une insatisfaction corporelle, d'un désir de modifier son poids et sa silhouette ou encore l'apparition de comportements compensatoires inappropriés (Currie, 2010). Dans ce contexte, l'absence de visée compétitive chez les sportifs de loisir pourrait agir comme facteur de protection relativement au développement d'une image corporelle négative et peut-être expliquer en partie les différences observées. Les résultats de l'étude actuelle mettent en relief le fait que, comparativement à des sportifs de niveau loisir, les sportifs de niveau intensif ressentent plus de pression concernant le jugement de l'entraîneur et des coéquipiers à faire un régime et perdre du poids. On peut présumer que, comparativement aux sportifs de niveau loisir, les sportifs de niveau intensif sont constamment en train de se comparer aux meilleurs dans leur discipline et ont bien peu l'occasion de se comparer favorablement. Selon 
la théorie de la comparaison sociale, ces comparaisons défavorables pourraient rendre les jeunes sportifs de niveau intensif plus vulnérables sur le plan de l'estime globale de soi et engendrer une perception physique de soi plus faible (Corning \& Gondoli, 2012). Ces mêmes jeunes, de par le fait qu'ils évoluent dans des milieux où l'apparence et le poids occupent une place prépondérante dans le discours des entraîneurs et des coéquipiers, deviendraient encore plus à risque de vivre une détresse psychologique relativement à leur poids et la performance sportive qui en découle et d'éventuellement développer un TCA (Corning \& Gondoli, 2012).

Contrairement aux résultats avancés par Haase et Prapavessis (2001), les résultats de l'étude actuelle montrent que, dans notre échantillon, les sportifs de niveau intensif éprouvent plus d'anxiété physique sociale que les sportifs de niveau loisir. $\mathrm{Si}$, d'une part, chez les adolescents l'investissement dans une pratique sportive peut être un moyen de chercher à modifier son apparence afin de diminuer l'anxiété physique sociale (Petrie \& Greenleaf, 2012), mais que, d'autre part, la pratique sportive est liée à une plus grande anxiété physique sociale (Prapavessis, Grove, \& Eklund, 2004), il est possible de croire que des sportifs de niveau intensif ressentent plus d'anxiété physique sociale que des sportifs de niveau loisir. II semble également qu'un volume et une fréquence plus élevés de pratique sportive dans une discipline sportive très axée sur le corps, comme la gymnastique ou la nage synchronisée, apportent davantage de préoccupations physiques tant sur le plan technique qu'esthétique, pouvant ainsi engendrer une plus grande anxiété physique sociale chez le sportif (de Bruin et al., 2011; Kong \& Harris, 2014). De la même façon, les sportifs qui pratiquent de façon intensive ressentent plus de pression pour faire un régime et perdre du poids que les sportifs qui pratiquent en loisir et, pour eux, ces pressions proviennent de sources plus nombreuses. Cette pression pourrait être liée à la recherche de performance physique et sportive (Byrne \& McLean, 2002), en tentant d'atteindre un corps idéal (Martinsen et al., 2010). En effet, dans la culture sportive, il est couramment admis qu'un corps longiligne et l'obtention d'un ratio poids/performance le plus bas possible permettent de meilleurs résultats sportifs (Currie, 2010). Par conséquent il est possible de présumer que les sportifs de niveau intensif pourraient percevoir plus de pression pour répondre à ce critère que les sportifs de niveau loisir, et ce, que ces pressions soient réelles ou non. Par ailleurs, les deux groupes de pratiquants rapportent une pression perçue pour être mince, perdre du poids et faire un régime similaire de la part de leurs parents, ce qui pourrait refléter une intolérance plus générale relative au surpoids ou encore une insatisfaction corporelle normative, indépendamment du niveau de pratique sportive (Tantleff-Dunn, Barnes, \& Larose, 2011). Cette pression également ressentie par rapport à la minceur pourrait aussi être le reflet d'un surinvestissement des parents dans la réussite sportive de leur enfant 
(Puper-Ouakil, Michel, Baup, \& Mouren-Siméoni, 2002) ou même d'un désir d'influencer positivement ses performances sportives en le motivant à contrôler son poids et à être mince.

\section{Limites et perspectives}

L'étude actuelle comporte certaines limites qu'il importe de mentionner. Tout d'abord, il s'agit de données autorapportées, elles peuvent donc être moins précises que des données obtenues en utilisant des méthodes plus objectives telles que les entretiens cliniques ou l'observation directe. Ensuite, les instruments de mesure utilisés ont été validés auprès d'un public adolescent, mais non auprès d'un public sportif, ce qui peut causer des biais de réponse. L'âge des participants peut également être une limite à cette étude, car d'un point de vue développemental, les adolescents de 13 et 20 ans sont très différents. Cependant, les caractéristiques des TCA chez le sportif de 13 et 20 ans sont similaires et les ANCOVAs réalisées ont permis de contrôler la variable âge. Notons aussi que cette étude a été menée dans un contexte culturel spécifique, soit celui de la pratique d'un sport de haut niveau en France, ce qui peut en limiter la possibilité de généralisation à d'autres populations de sportifs, bien qu'une partie de nos résultats corroborent les résultats d'études internationales antérieures. Enfin, un devis de recherche longitudinal semble nécessaire afin de vérifier les effets à moyen terme sur le risque de développer un TCA chez les adolescents qui pratiquent un sport selon une fréquence et une intensité permettant de les situer à différents endroits sur ce continuum. Ce devis permettrait également de vérifier si les symptômes alimentaires les plus prévalents, comme les régimes, évoluent avec le temps vers des crises de suralimentation et d'autres formes de stratégies de contrôle du poids telles que les vomissements et l'abus de laxatifs ou de diurétiques.

\section{CONCLUSION}

Les résultats de cette étude permettent ainsi de dégager un portrait global de certaines caractéristiques comportementales et psychosociales chez un groupe de sportifs engagés dans des pratiques à risque pour les TCA. II est alors possible de dresser un profil de sportifs selon leur niveau de pratique. Les résultats suggèrent que, même s'ils n'ont pas nécessairement de diagnostic de TCA, un continuum des ACAl existe chez les sportifs : ceux pratiquant à un niveau intensif étant plus susceptibles d'adopter des comportements de contrôle du poids et d'être insatisfaits de leur corps que ceux qui pratiquent à un niveau de loisir. Les sportifs qui pratiquent de façon intensive présentent par ailleurs un niveau d'anxiété physique sociale plus élevée et ils perçoivent plus de pression pour faire un régime ou perdre du poids de la part de leurs entraîneurs et coéquipiers. Ainsi, il semble probable que l'activité physique et sportive 
pratiquée dans un contexte de performance puisse favoriser l'émergence d'ACAl. Malheureusement, bien que les TCA constituent une préoccupation de plus en plus présente dans le contexte sportif (Bonci et al., 2008; Tan, Bloodworth, McNamee, \& Hewitt, 2014), la spécificité des TCA chez le sportif peut représenter un défi pour les intervenants. En effet, ces derniers sont souvent peu informés et inconfortables avec le sujet (Torres McGehee, Leaver-Dunn, Green, Bishop, Leeper, \& Richardson, 2011). Pour leur part, les intervenants et psychologues en contexte clinique traditionnellement habitués à prescrire une contre-indication à la pratique sportive lorsqu'un TCA est soupçonné, qui est alors assimilée à de l'hyperactivité, peuvent être, à leur tour, mal à l'aise d'intervenir auprès de sportifs de niveau intensif chez qui la pratique sportive est indissociable de leur style de vie, voire de leur personnalité (Miller \& Jome, 2010). II semble donc nécessaire que les intervenants de tous champs possèdent une connaissance précise des caractéristiques personnelles et liées à la pratique sportive, pouvant faire évoluer le sportif sur le continuum des ACAI, afin de pouvoir procéder à une évaluation appropriée permettant par la suite de proposer des pistes d'intervention adaptées aux réalités de la population sportive.

\section{RÉFÉRENCES}

Ackard, D. M., Fulkerson, J. A., \& Neumark-Sztainer, D. (2007). Prevalence and utility of DSM-IV eating disorder diagnostic criteria among youth. International Journal of Eating Disorders, 40(5), 409-417.

American Psychiatric Association (2014). DSM-5 -Diagnostic and statistical manual of mental disorders. Arlington, VA : American Psychiatric Association.

Beals, K. A. (2004). Disordered eating among athletes: A comprehensive guide for health professionals. Champaign, IL : Human Kinetics.

Bonci, C. M., Bonci, L. J., Granger, L. R., Johnson, C. L., Malina, R. M., Mine, L. W., ... Vanderbunt, E. M. (2008). National athletic trainers' association position statement: Preventing, detecting, and managing disordered eating in athletes. Journal of Athletic Training, 43(1), 80-108.

Bratland-Sanda, S., \& Sundgot-Borgen, J. (2013). Eating disorders in athletes : Overview of prevalence, risk factors and recommendations for prevention and treatment. European Journal of Sport Science, 3(5), 499-508.

Brunet, J, \& Sabiston, C. M. (2009). Social physique anxiety and physical activity: A selfdetermination theory perspective. Psychology of Sport and Exercise, 10(3), 329-335.

Byrne, S., \& McLean, N. (2002). Elite athletes: Effects of pressure to be thin. Journal of Science and Medicine in Sport, 5(2), 80-94.

Callahan, S., Rousseau, A., Knotter, A., Bru, V., Danel, M., Cueto, C., ... Chabrol, H. (2003). Les troubles alimentaires: présentation d'un outil de diagnostic et résultats d'une étude épidémiologique chez les adolescents. Encéphale, 29(1), 239-247.

Chatterton, J. M., \& Petrie, T. A. (2013). Prevalence of disordered eating and pathogenic weight control behaviors among male collegiate athletes. Eating Disorders, 21(4), 328341.

Cole, T. J., Bellizzi, M. C., Flegal, K. M., \& Dietz, W. H. (2000). Establishing a standard definition for child overweight and obesity worldwide: International survey. British Medical Journal, 320(7244), 1240-1243.

Cole, T. J., Flegal, K. M., Nicholls, D., \& Jackson, A. A. (2007). Body mass index cut offs to define thinness in children and adolescents: International survey. British Medical Journal, 335(7612), 194-197. 
Corning, A. F., \& Gondoli, D. M. (2012). Who is more likely to fat talk? A social comparison perspective. Body Image, 9(4), 528-531.

Cox, R. H. (2007). Sport psychology: Concepts and applications. New York, NY : McGrrawHill.

Currie, A. (2010). Sport and eating disorders: Understanding and managing the risks. Asian Journal of Sports Medicine, 1(2), 63-68.

Darcy, A. M., Hardy, K. K., Lock, J., Hill, K. B., \& Peebles, R. (2013). The eating examination questionnaire (EDE-Q) among university men and women at different levels of athleticism. Eating Behaviors, 14(3), 378-381.

de Bruin, A. P., Oudejans, R. R. D., Bakker, F. C., \& Woertman, L. (2011). Contextual body image and athletes' disordered eating: The contribution of athletic body image to disorderd eating in high performance women athletes. European Eating Disorders Review, 19(3), 201-215.

Deimel, J. F., \& Dunlap, B. J. (2012). The female athlete triad. Clinics in Sports Medicine, $31(2), 247-254$.

Fairburn, C. G. (2008). Cognitive behavior therapy and eating disorders. New York, NY: Guilford Press.

Fortes, L. de S., Kakeshita, I. S., Almeida, S. S., Gomes, A. R., \& Ferreira, M. E. C. (2014). Eating behaviors in youths: A comparison between female and male athletes and nonathletes. Scandinavian Journal of Medicine \& Science in Sports, 24(1), e62-e68.

Fox, K. R. (2000). Self-esteem, self-perception and exercise. International Journal of Sport Psychology, 31(2), 228-240.

Gapin, J. I., \& Kearns, B. (2013). Assessing prevalence of eating disorders and eating disorders symptoms among lightweight and open weight collegiate rowers. Journal of Clinical Sport Psychology, 7(3), 198-214.

Garner, D. M., Olmstead, M. P., \& Polivy, J. (1983). Development and validation of a multidimensional eating disorder inventory for anorexia nervosa and bulimia. International Journal of Eating Disorders, 2(2), 15-34.

Grandmontagne, A. G., \& Fernández, A. R. (2002). Eating disorders, sport practice and physical self-concept in adolescents. Actas Españolas de Psiquiatria, 32(1), 29-36.

Greenleaf, C., Petrie, T. A., Carter, J., \& Reel, J. J. (2009). Female collegial athletes : Prevalence of eating disorders and disordered eating behaviors. Journal of American College Health, 57(5), 489-496.

Grieve, F. G. (2007). A conceptual model of factors contributing to the development of muscle dysmorphia. Eating Disorders, 15(1), 63-80.

Haase, A. M., \& Prapavessis, H. (2001). Social physique anxiety and eating attitudes in female athletic and non-athletic groups. Journal of Science and Medicine in Sport, 4(4), 396-405.

Hart, E., Leary, M. R., \& Rejeski, W. J. (1989). The measurement of social physique anxiety. Journal of Sport \& Exercise Psychology, 11(1), 94-104.

Hoek, H. W. (2006). Incidence, prevalence and mortality of anorexia nervosa and other eating disorders. Current Opinion in Psychiatry, 19(4), 389-394.

Hua, L. V., \& Braddock, J. H. (2008). School sports and adolescent steroid use: National trends and race-ethnic variations. Challenge, 14(2), 29-41.

Johnson, M. D. (1994) Disordered eating in active and athletic women. Clinics in Sports Medicine, 13(2), 355-369.

Kong, P., \& Harris, L. M. (2014). The sporting body: body image and eating disorders symptomatology among female athletes from leanness focused and nonleanness focused sports. The Journal of Psychology, 149(2), 141-160.

Koszewski, W., Chopak J. S., \& Buxton, B. P. (1997). Risk factors for disordered eating in athletes. Athletic Therapy Today, 2(2), 7-11.

Lanfranchi, M.-C., Maïano, C., Morin, A. J. S., \& Therme, P. (2014). Prevalence and sportrelated predictors of disturbed eating attitudes and behaviors : Moderating effects of sex and age. Scandinavian Journal of Medicine \& Science in Sport, 24(4), 622-633.

Leung, F., Geller, J., \& Katzman, M. (1996). Issues and concerns associated with different risk models for eating disorders. International Journal of Eating Disorders, 19(3), 249256. 
Maïano, C., Morin, A, J, S., Eklund, R. C., Monthuy-Blanc, J., Garbarino, J.-M., \& Stephan, Y. (2010). Construct validity of the Social Physique Anxiety Scale in a french adolescent Sample. Journal of Personality Assessment, 92(1), 53-62.

Maïano. C., Morin, A. J. S., Monthuy-Blanc, J., Garbarino, J.-M., \& Stephan, Y. (2009). Eating disorders inventory : Assessment of its construct validity in a nonclinical french sample of adolescents. Journal of Psychopathology and Behavioral Assessment, 31(4), 387-404.

Maïano, C., Morin, A. J. S., Ninot, G., Monthuy-Blanc, J., Stephan, Y., Florent, J.-F., \& Vallée, P. (2008). A short and very short form of the physical self-inventory for adolescents : Development and factor validity. Psychology of Sport and Exercise, 9(6), 830-847.

Martinsen, M., Bratland-Sanda, S., Eriksson, A. K., \& Sundgot-Borgen, J. (2010). Dieting to win or to be thin? A study of dieting and disordered eating among adolescent elite athletes and non-athlete controls. British Journal of Sports Medicine, 44(1), 70-76.

Martinsen, M., \& Sundgot-Borgen, J. (2013). Higher prevalence of eating disorders among adolescent elite athletes than controls. Medicine \& Science in Sports \& Exercise, 45(6), 1188-1197.

Miller, D. N., \& Jome, L. M. (2010). School psychologist and the secret illness : Perceived knowledge, role preferences, and training needs regarding the prevention and treatment of interalizing disorders. School Psychology International, 31(5), 509-520.

Mintz, L. B., O'Halloran, M. S., Mulholland, A. M., \& Schneider, P. A. (1997). Questionnaire for eating disorders diagnosis: Reliability and validity of operationalizing DSM-IV criteria into a self-report format. Journal of Counseling Psychology, 44(1), 63-79.

Monthuy-Blanc, J., \& Bonanséa, M. (2014) Eating disorders in athletes. In M. Probst \& A. Carraro (Éds), Mental health and physical activity from a practice oriented perspective. (p. 117-121). Milan, Italie : Edi Ermes.

Monthuy-Blanc, J., Bonanséa, M., Maïano, C., Therme, P., Lanfranchi, M-C., Pruvost, J., \& Serra, J.-M. (2010). Guide de recommandations: prévention des troubles $d u$ comportement alimentaire des sportifs à destination des professionnels et/ou des bénévoles du sport et de la santé. Paris : Fédération française d'athlétisme.

Monthuy-Blanc, J., Maïano, C., Morin, A. J. S., \& Stephan, Y. (2012). Physical self-concept and disturbed eating attitudes and behaviors in french athlete and non-athlete adolescent girls : Direct and indirect relations. Body Image, 9(3), 373-380.

Mosley, P. E. (2009). Bigorexia: Bodybuilding and muscle dysmorphia. European Eating Disorders Review, 17(3), 191-198.

Peden, J., Stiles, B. L., Vandehey, M., \& Diekhoff, G. (2008). The effects of external pressures and competitiveness on characteristics of eating disorders and body dissatisfaction. Journal of Sport and Sciences Issues, 32(4), 415-429.

Petrie, T. A., \& Greenleaf, C. (2012). Eating disorders in sport. In S. M. Murphy (Éd.), The Oxford handbook of sport and performance psychology (p.635-659). New York, NY: Oxford University Press.

Petrie, T. A., Greenleaf, C., Reel, J., \& Carter, J. (2008). Prevalence of eating disorders and disordered eating behavior among male collegiate athlete. Psychology of Men and Masculinity, 9(4), 267-277.

Prapavessis, H., Grove, J. R., \& Eklund, R. C. (2004). Self-presentational issues in competition and sport. Journal of Applied Sports Psychology, 16(1), 19-40.

Puper-Ouakil, D., Michel, G., Baup, N., \& Mouren-Siméoni, M.-C. (2002). Aspects psychopathologiques de l'exercice intensif chez l'enfant et l'adolescent : mise au point à partir d'une situation clinique. Annales médico-psychologiques, 160(8), 543-549.

Smolak, L., Murnen, S. K., \& Ruble, A. E. (2010). Female athletes and eating problems : A meta-analysis. International Journal of Eating Disorders, 27(4), 371-380.

Stice, E., \& Agras, W. S. (1998). Predicting onset and cessation of bulimic behaviors during adolescence : A longitudinal Gouping analysis. Behavior Therapy, 29(2), 257-276.

Sundgot-Borgen, J. (1994). Risk and trigger factors for the development of eating disorders in female elite athletes. Medicine \& Science in Sports and Exercise, 26(4), 414-419.

Sundgot-Borgen. J, Meyer, N. L., Lohman, T. G., Ackland, T. R., Maughan, R. J., Stewart, A. D., \& Müller, W. (2013). How to minimize the health risks to athletes who compete in weight- sensitive sports review and position statement on behalf of the ad hoc research 
working group on body composition, health and performance, under the auspices of the IOC Medical Commission. British Journal of Sports and Medicine, 47(16), 1012-1022.

Sundgot-Borgen, J., \& Torstveit, M. K. (2004). Prevalence of eating disorders in elite athletes is higher than in the general population. Clinical Journal of Sport Medicine, 14(1), 25-32.

Sundgot-borden, J., \& Torstveit, M. K. (2010). Aspects of disordered eating continuum in elite high-intensity sports. Scandinavian Journal of Medicine \& Sciences in Sports, 20(s2), 112-121.

Tan, J., Bloodworth, A., McNamee, M., \& Hewitt, J. (2014). Investigating eating disorders in elite gymnasts: Conceptual, ethical and methodological issues. European Journal of Sport Science, 14(1), 60-68.

Tantleff-Dunn, S., Barnes, R. D., \& Larose, J. G. (2011). It's not just a "woman thing" : The current state of normative discontent. Eating Disorders, 19(5), 392-402.

Thompson, A. M., \& Chad, K. E. (2002). The relationship of social physique anxiety to risk for developing an eating disorder in young females. Journal of Adolescent Health, 31(2), 183-189.

Toro, J., Galilea, B., Martinez-Mallén, E., Salamero, M., Capdevilla, L., Mari, J., ... Toro, E. (2005). Eating disorders in spanish female athletes. International Journal of Sports Medicine, 26(8), 693-700.

Torres McGehee, T. M., Leaver-Dunn, D., Green, J. M., Bishop, P. A., Leeper, J. D., \& Richardson, M. T. (2011). Knowledge of eating disorders among collegiate administrators, coaches, and auxilliary dancers. Perceptual and Motors Skills, 112(3), 951-958.

Torstveit, M. K., Rosenvinge, J. H., \& Sundgot-Borgen, J. (2008). Prevalence of eating disorders and the predictive power of risk models in female elite athletes : A controlled study. Scandinavian Journal of Medicine \& Science in Sports, 18(1), 108-118.

Turgeon, M.-E. K., Meilleur, D., \& Blondin, S. (2015). Évaluation des attitudes et des comportements alimentaires: comparaison entre un groupe d'adolescentes athlètes pratiquant un sport esthétique et un groupe témoin. Neuropsychiatrie de l'enfance et de l'adolescence, 63(3), 175-182.

Tylka, T. L., \& Subich, L. M. (2003). Revisiting the latent structure of eating disorders: taxometric analyses with non behavioral indicators. Journal of Counseling Psychology, $50(3), 276-286$.

\section{RÉSUMÉ}

L'objectif de cette étude est de comparer les attitudes et comportements alimentaires inappropriés (ACAI) ainsi que les caractéristiques psychosociales - anxiété physique sociale, perceptions du soi physique, pression perçue pour être mince - entre deux niveaux de pratique sportive (intensive et de loisir). Cent quarante-six adolescents français ont rempli un ensemble de questionnaires. Les résultats indiquent que, comparativement aux sportifs qui s'entraînent en loisir, les sportifs de niveau intensif sont plus nombreux à avoir recours à des ACAl, ils ressentent également plus d'insatisfaction corporelle et d'anxiété physique sociale, ils sont moins satisfaits de leur apparence physique et ressentent plus de pression pour mincir.

\section{MOTS CLÉS}

niveau de pratique sportive; comportements alimentaires inappropriés; anxiété physique sociale; insatisfaction corporelle; pression sociale; perceptions du soi physique;

\section{ABSTRACT}

The aim of this study is to compare inappropriate attitude and eating behaviors (IAEB) and psychosocial characteristics - physical social anxiety, physical self-perceptions, perceived pressure to be thin - between two levels of sport practice (intensive and recreational). A set of 
questionnaires was completed by 146 French adolescents. Results indicate that compared to recreational athletes, those training at an intensive level are more likely to resort to IAEB. Moreover, elite athletes also suffer more from body dissatisfaction and social physical anxiety, are less satisfied with their physical appearance, and are feeling more pressure to be thinner.

\section{KEY WORD}

sport practice level; inappropriate eating behavior; social physical anxiety; body

dissatisfaction; social pressure; physical self-perceptions 